\title{
Comment on "Instability studies at the CERN Proton Synchrotron during transition crossing"
}

\author{
A. Burov \\ Fermilab, P.O. Box 500, Batavia, Illinois 60510-5011, USA
}

(Received 10 January 2019; published 19 June 2019)

\begin{abstract}
In the article of Migliorati, Aumon, Koukovini-Platia, Huschauer, Metral, Sterbini and Wang [Phys. Rev. Accel. Beams 21, 120101 (2018)], hereafter the Article and the Authors, the long-standing qualification of the PS instability as the transverse mode-coupling instability, or TMCI, is not mentioned, while a model of the beam breakup (BBU) is discussed. Do the observations and modeling of the Article refute the longstanding idea that the PS instability is the TMCI?
\end{abstract}

DOI: $10.1103 /$ PhysRevAccelBeams.22.068001

For decades, it was assumed, and not just at CERN, that when the coherent tune shift of a bunch is comparable with the synchrotron tune, the bunch becomes unstable due to the transverse mode coupling instability (TMCI), more or less independently of the space charge (SC). This assumption appeared to be supported by measurements and simulations, so it seemed to be well justified to classify the transverse instabilities in CERN PS and SPS rings as TMCI. This conviction was expressed by CERN-associated authors many times when the instabilities were discussed, at least since the year 2000, when Ref. [4] of [1] (the Article) was published. In that EPAC 2000 paper, the following was written about the PS instability:

"... this instability is in fact equivalent to the Transverse Mode Coupling instability of Kohaupt in terms of coupling of head-tail modes... However, the mode coupling formalism is adequate only when the intensity threshold is approached from below (i.e., when the coherent betatron frequency shift becomes comparable to the incoherent synchrotron frequency). When fast beam losses occur within a fraction of the synchrotron period, i.e., for the case well above threshold, the concept of head-tail modes loses its meaning. In this case, it seems more appropriate to describe the interaction between the beam and its surroundings in terms of beam breakup".

The same ideas about this instability were expressed in a recent publication [2]:

"As the bunch intensity increases, the different head-tail modes can no longer be treated separately. In this regime, the wake fields couple the modes together and a wave

\footnotetext{
*burov@fnal.gov

Published by the American Physical Society under the terms of the Creative Commons Attribution 4.0 International license. Further distribution of this work must maintain attribution to the author(s) and the published article's title, journal citation, and DOI.
}

pattern travelling along the bunch is created [see e.g., Fig. 14 (lower)]: this is the Transverse Mode Coupling Instability (TMCI)".

It is worth mentioning that there was a purely theoretical objection to the TMCI-explanation of the PS and SPS instabilities: with the SC tune shifts as high as they are in those machines, TMCI should not be possible. Vanishing of TMCI at high SC was first shown by M. Blaskiewicz in 1998 for some special cases [3], and later was confirmed by several authors [4-6] for practically all possible situations. In an apparent contradiction to this theoretical conclusion, the instabilities were observed at PS and SPS, approximately at the same parameters where TMCI at no SC should be. The situation looked like SC has no significant effect on TMCI.

A resolution of this conundrum was suggested by the author of this comment in summer of 2018 [7,8]; the first of these papers was recently published in this journal [9]. It was shown that while sufficiently strong SC indeed removes TMCI, it opens the door for a family of convective instabilities, for pretty much the same range of parameters.

The Article is not purely empirical. To explain the observations, at least to some degree, the Article suggests a specific BBU model, comparing its results with the PyHEADTAIL simulations, Fig. 18. The BBU code is described in details in the Appendix. At the same time, nothing at all is said about a possible role of the TMCI mechanism, which was used to explain the observations in many publications for the last twenty years. Do the observations and modeling of the Article refute the longstanding idea that the PS instability is the TMCI? Do they rather confirm it? Do they neither refute, nor confirm it? Apparently, we deal here with new physics, so the question is important.

Fermilab is operated by Fermi Research Alliance, LLC under Contract No. DE-AC02-07CH11359 with the United States Department of Energy. 
[1] M. Migliorati, S. Aumon, E. Koukovini-Platia, A. Huschauer, E. Métral, G. Sterbini, and N. Wang, Instability studies at the CERN Proton Synchrotron during transition crossing, Phys. Rev. Accel. Beams 21, 120101 (2018).

[2] E. Mtral et al., Beam instabilities in hadron synchrotrons, IEEE Trans. Nucl. Sci. 63, 1001 (2016).

[3] M. Blaskiewicz, Fast head-tail instability with space charge, Phys. Rev. ST Accel. Beams 1, 044201 (1998).

[4] A. Burov, Head-tail modes for strong space charge, Phys. Rev. ST Accel. Beams 12, 044202 (2009); Erratum, Phys. Rev. ST Accel. Beams 12, 109901(E) (2009).
[5] V. Balbekov, Threshold of transverse mode coupling instability with arbitrary space charge, Phys. Rev. Accel. Beams 20, 114401 (2017).

[6] T. Zolkin, A. Burov, and B. Pandey, Transverse modecoupling instability and space charge, Phys. Rev. Accel. Beams 21, 104201 (2018).

[7] A. Burov, Convective instabilities of bunched beams with space charge, arXiv:1807.04887.

[8] A. Burov, Core-Halo collective instabilities, arXiv:1808.08498.

[9] A. Burov, Convective instabilities of bunched beams with space charge, Phys. Rev. Accel. Beams 22, 034202 (2019). 